\title{
Cusack U.S Economy Equation
}

1641 Sandy Point Rd, Saint John, NB, Canada E2K 5E8, Canada

*Corresponding author: Cusack PTE, Independent Researcher, BSc E, DULE, 1641 Sandy Point Rd, Saint John, NB, Canada E2K 5E8, Canada, Tel: (506) 214-3313; E-mail: St-michael@hotmail.com

Received date: January 17, 2017, Accepted date: February 23, 2017, Published date: March 02, 2017

Copyright: (c) 2017 Cusack PTE. This is an open-access article distributed under the terms of the Creative Commons Attribution License, which permits unrestricted use, distribution, and reproduction in any medium, provided the original author and source are credited.

\section{Abstract}

Here is a paper that uses 11 variables in an equation that describes the US economy. It can be used to predict the peaks to crash and where it is in the economic cycle.

Keywords: Macroeconomics; Key economic indicators

\section{Introduction}

$\mathrm{GDP}=\mathrm{e}^{\wedge} \mathrm{x}$

Dow Jones Industrial Average $=\sin$

Standard and Poor ${ }^{`} 500$ Index $=\sin / \mathrm{e}^{\wedge} \mathrm{x}$

$\mathrm{CPI}=\mathrm{e}^{\wedge} \mathrm{x}$

Unemployment $=\sin$

Commodities Index

Oil linear

Gold Production $=$ constant

New Residential Construction=linear

Personal Income=linear

US Trade Balance $=$ sin

Global Stock Market $=\mathrm{e}^{\wedge} \mathrm{x}$ sin

Each one of these indicates is a sin, cos or exponential function.

$\mathrm{SO}$,

$\mathrm{y}=\mathrm{y}^{\prime}$

$\mathrm{y}=\mathrm{e}^{\wedge} \mathrm{x}$

$\mathrm{y}=\mathrm{y}^{\prime}$

$\mathrm{y}=\sin \mathrm{x}+\mathrm{C} 1=\cos \mathrm{x}+\mathrm{C} 2$

$\mathrm{x}=1, \mathrm{Y}=0.8415 \mathrm{cf} 0.86$

$0.8415+\mathrm{C} 3=\mathrm{e}^{\wedge} \mathrm{x}$

Boundary condition

$\mathrm{x}=1$

$\mathrm{C} 3=\mathrm{c}=1.8768$

$\mathrm{Y}=\mathrm{e}^{\wedge} \mathrm{x}-1.8768$

Now for the Fourier series:

$\mathrm{y}=\mathrm{e}^{\wedge} \mathrm{x}-1.8768$ $\mathrm{y}=\mathrm{a} \sin \mathrm{x}+\mathrm{b} \cos \mathrm{x}$

$a \sin \mathrm{x}+\mathrm{b} \cos \mathrm{x}=\mathrm{e}^{\wedge} \mathrm{x}-1.8768$

$\mathrm{a}(0.86)+\mathrm{b}(0.86)=0.8415$

$(a+b)=1$

$\mathrm{a}=1-\mathrm{b}$

$(1-b) \sin x+b \cos x=e^{\wedge} x-1.8768$

$0.86-b(0.86)-b(0.86)=e^{\wedge} 1-1.8768$

$\mathrm{y}=\mathrm{e}^{\wedge} 1-1.8768$

$\mathrm{Y}=0.8415$

For a full cycle:

$1 / 2 \mathrm{Pi}=0.1592$

$0.1592 \mathrm{x}=6.28$

$\mathrm{x}=39.467$

So from peak to trough $=39.4627(2)=78.93$ years

October $29,1929+78.93=2007.9$

November 4, 2007 Peak to crash

$\mathrm{GDP}=\mathrm{e}^{\wedge} \mathrm{x}$

Dow Jones Industrial Average $=\sin$

Standard and Poor`s 500 Index $=\sin / \mathrm{e}^{\wedge} \mathrm{x}$

$\mathrm{CPI}=\mathrm{e}^{\wedge} \mathrm{x}$

Unemployment $=\sin$

Commodities Index

Oil linear

Gold Production=constant

New Residential Construction=linear

Personal Income=linear

US Trade Balance $=$ sin

Global Stock Market $=\mathrm{e}^{\wedge} \mathrm{x}$ sin 
S\&P500 $=-(G D P * C P I) e^{\wedge} x+0.8415\left(\sin \mathrm{TDJIA}^{\star} \sin \mathrm{T}\right.$ UnEmploy ${ }^{*} \sin$ $\mathrm{T}$ US Trade Balance) $\sin \mathrm{x}+\mathrm{x}($ oil production + mean house price + Personal Incxome)

For Annum $1990 \mathrm{X}=1$ Maximum:

$400=\left(7113^{*} 391.4\right) \mathrm{e}^{\wedge} \mathrm{Y}^{\star} 0.7460+(30,000+92,000+4878.6) \mathrm{X} / 1000$

$\mathrm{E}^{\wedge} \mathrm{Y}=0.1315 \mathrm{x}=0.202=\mathrm{Y}$ Output Energy

S\&P500=-(GDP*CPI) $\mathrm{e}^{\wedge} \mathrm{Y}+0.8415\left(\sin \mathrm{TDJIA}^{*} \sin \mathrm{T}\right.$ UnEmploy ${ }^{\star} \sin$

$\mathrm{T}$ US Trade Balance) sin $\mathrm{X}+\mathrm{x}$ (oil production + mean house price + Personal Incxome)

$400=\left(7113^{\star} 391.4\right) \mathrm{e}^{\wedge} \mathrm{Y}^{\star} 0.7460+(30,000+92,000+4878.6) \mathrm{X} / 1000$

$\mathrm{e}^{\wedge} \mathrm{Y}=0.1315 \mathrm{x}=0.202=\mathrm{Y}$ Output Energy

Since $\mathrm{Y}=\mathrm{e} 1-1.8768$

$\mathrm{Y}=2.71828-1.8768$

$0.202=2.71828-1.8768(\mathrm{Pi} / 2)=\mathrm{e}^{\wedge} \mathrm{Y}$

$\mathrm{Pi} / 2 * \mathrm{Y}=\mathrm{e}^{\wedge} \mathrm{Y}=\mathrm{Pi}$

$\mathrm{Y}=\mathrm{Ln} \mathrm{Pi}$

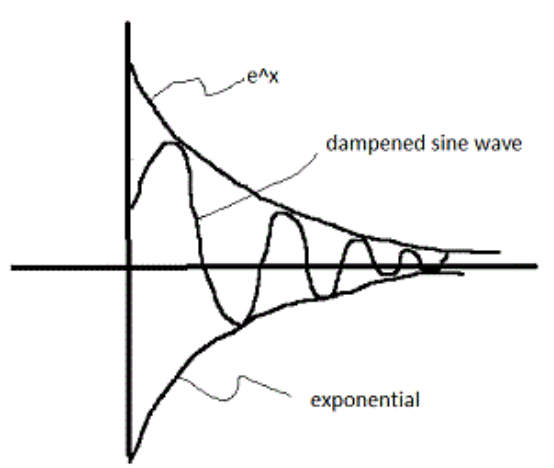

Figure 1: Dampened cosine curve.
$\mathrm{Y}=\mathrm{e}^{\wedge} 1-1.8768$ Refer to Figure 1.

$\mathrm{Y}=0.8415$

But $\mathrm{Y}=0.202$ (dampened sine wave)

So,

$\mathrm{Y}=\mathrm{e}^{\wedge} 0.1315=1.1405$

$\mathrm{y}=1 / \mathrm{y}$

$\operatorname{Ln} x=1 / x$

\section{Conclusion}

$y=y^{\prime}$ for the economy. 